\title{
Imaging of the Acute Scrotum: Is There a Place for Contrast-Enhanced Ultrasonography?
}

\author{
H. Moschouris, K. Stamatiou, E. Lampropoulou, D. Kalikis, D. Matsaidonis \\ Departments of Radiology (HM, EL, DK DM) and Urology (KE), General Hospital of Pireas Tzaneio, \\ Pireas, Greece
}

\begin{abstract}
Purpose: To present and evaluate the findings of contrast-enhanced ultrasonography (CEUS) in typical cases of acute painful scrotum.

Materials and Methods: Nineteen patients aged from 19 to 61 years old were included in the study. All patients underwent grey-scale and color Doppler ultrasonography (US) of the scrotum, followed by imaging after i.v. administration of 2.4 $\mathrm{mL}$ of a second generation ultrasound contrast agent (microbubbles of sulphur hexafluoride). A dedicated, contrast-sensitive technique was used (Contrast Tissue Imaging - CnTI). The diagnosis was confirmed surgically in 6 cases while in the remaining 8 cases it was based on the combination of clinical, imaging and laboratory findings.

Results: The final diagnosis was testicular torsion $(\mathrm{n}=4)$, epididymitis $(\mathrm{n}=2$, one of the cases complicated by abscess), testicular abscess $(n=1)$, scrotal abscess $(n=1)$, testicular trauma of varying severity $(n=6)$. Five out of 19 cases were true negatives: neither clinical examination nor laboratory tests revealed any pathology. CEUS showed complete lack of enhancement in all cases of torsion, permitting a rapid and definitive diagnosis. In the cases of infection complicated by abscesses, CEUS delineated the lesions much better than the combination of B-mode/Color Doppler US. The severely traumatized testicles showed minimal, inhomogeneous or patchy enhancement, while cases of minor trauma showed no significant enhancement defects. Hematomas were presented as non-enhancing lesions.

Conclusion: Generally, there was no advantage over Doppler US as has been previously shown. However, CEUS can be used supplementary to traditional Doppler US in the investigation of blunt testicular trauma especially when there is uncertainty in diagnosis after appropriate clinical and radiographic evaluations occurs. Further studies are required to clearly define the indications of this method.
\end{abstract}

Key words: testis; epididymis; torsion; trauma; color Doppler; ultrasound

Int Braz J Urol. 2009; 35: 692-705

\section{INTRODUCTION}

Due to the great efficacy of the existing facilities of modern ultrasonography (US) such as high frequency transducers, color and power Doppler, ultrasonography is considered the imaging modality of choice for evaluating acute and non acute scrotal disease $(1,2)$. Many of these disease processes, in- cluding testicular torsion, epididymo-orchitis, and intratesticular tumor, produce the common symptom of pain at presentation, and differentiation of these conditions and disorders is important in determining the appropriate treatment. US with a high-frequency transducer helps to better characterize intra-scrotal lesions, and in many instances the findings suggest diagnoses that are more specific. High-frequency US 
in its present state can be useful to identify certain benign intratesticular lesions, resulting in testessparing surgery (3). Nevertheless, a percentage of inconclusive sonograms -often causing medico-legal problems- are still reported in the literature (4).

Imaging modalities such as scintigraphy and magnetic resonance imaging (MRI) of the scrotum, which can be applied when ultrasonography proves inadequate, could provide a more accurate diagnosis in the evaluation of testicular perfusion than color Doppler US $(5,6)$. In particular MRI offers useful, and in some cases decisive, information, as it is capable of revealing unexpected findings and elucidating complex aspects. Additionally, MRI helps improve patient management, with an overall reduction in costs (5). Unfortunately, being specialized, relatively expensive and not always available it is not routinely used for the evaluation of acute painful scrotum but remains an ideal choice for second-line investigation.

From the view point that US is more convenient and easier to be performed in the emergency clinical settings than MRI, a contrast-enhanced ultrasound study would be the ideal tool in the assessment of testicular perfusion in patients with acute scrotal symptoms.

Under those circumstances, the investigation of the role of contrast-enhanced ultrasonography (CEUS) as a practical complementary tool in the study of the acute painful scrotum is worth exploring. To our knowledge, to date no similar CEUS study focusing exclusively on representative urgent, painful situations of acute painful scrotum has been reported in the literature.. Only two prior studies using this method have been previously performed in very small numbers of patients, and no advantage over Doppler US has been shown $(7,8)$. In contrast, a recent experimental study by Liang et al. demonstrated that CEUS was superior to color Doppler ultrasound in evaluating the perfusion of testicular ischemia (9).

The present study was carried out to illustrate the findings of CEUS in a variety of cases of acute scrotal pathology in order to evaluate its possible role in the investigation of painful scrotum. Our objective was also to analyze the advantages and limitations of this method and suggest potential indications.

\section{MATERIALS AND METHODS}

This controlled prospective study was carried-out at the General Hospital of Pireas, "Tzaneio" (Pireas, Greece) from April 2008 to November 2008. The study group included 20 consecutive cases with acute painful scrotum who presented at the outpatient department of our hospital. The exclusion criteria were a previous surgery of the testis, which may confuse the radiological image, and history of allergic reaction to any contrast agent. Only one out of the 20 patients had a previous surgery of the testis. The remaining nineteen patients who fulfilled the inclusion criteria were initially investigated with conventional scrotum ultrasonography and color and power Doppler.. CEUS was performed immediately afterwards. Both the basic examination and CEUS were performed with an Esaote Big GPX (Esaote, Genoa, Italy) ultrasound device in the radiology department by two experienced ultrasonographers.

A $2.4 \mathrm{~mL}$ of a second generation ultrasound contrast agent (microbubbles of sulphur hexafluoride, SonoVue, Bracco, Milan Italy) were injected as a bolus in the forearm vein followed by $5 \mathrm{~mL}$ of normal saline. Both the affected and the normal side of the scrotum were imaged with a contrast-specific technique (Contrast Tuned Imaging- CnTI) combined with low mechanical index $(\mathrm{MI}=0.07-0.08)$ in order to obtain a satisfactory shading of the testicle for at least 2.5-3 minutes. A high acoustic power "flash" causing instant disruption of the microtubules was applied when deemed necessary by the sonographer. The examination was performed with a linear 5-7.5 $\mathrm{MHz}$ transducer. Patients with profound scrotal swelling were also studied with a convex $2.5-5 \mathrm{MHz}$ transducer. Those with pathological ultrasound and/or clinical findings were further evaluated and treated. For study purposes, a uniform registration form was used for recording findings of Doppler US and CEUS from each patient. Representative images from the baseline examination and the entire CEUS examination were stored as digital archives in the computer database. In all cases, the reported findings of CEUS were correlated with those of the grey-scale and colored Doppler. Comparison with the normal side made the identification of pathologic enhancement easier. Final diagnosis was based upon clinical and labora- 
tory examination and/or surgical exploration. Upon the final evaluation of the CEUS technique, images were examined by separate examiners blinded to the other's results. Finally, the recorded images were reviewed to reach a consensus.

The study was approved by the locally appointed Ethics Committee, and all informed patients gave their written signed consent. The research protocol was performed in accordance with the International Conference on Harmonisation Guideline for Good Clinical Practice (1996), which represents the international ethical and scientific quality standard for designing, conducting, recording, and reporting trials that involve participation of human subjects.

\section{RESULTS}

Five out of 19 cases were true negatives: neither clinical examination nor laboratory testing revealed any pathology. Similarly, both the basic examination and CEUS were normal Therefore, they were excluded from the study. Contrast-enhanced examination was successfully completed and its quality was considered adequate for inclusion in the study in the remaining 14 patients with abnormal ultrasound findings. No patient suffered any adverse reactions despite the fact almost all patients were not on an empty stomach. In all the 14 cases both the baseline ultrasound examination and CEUS diagnosis was in accordance with that of clinical/laboratory examination and surgery/pathology findings.

The definite diagnosis was confirmed upon immediate surgical exploration in 6 cases: testicular rupture $(n=2)$, testicular torsion $(n=4)$. In the remaining 8 cases, diagnosis was based on the combination of clinical, imaging and laboratory findings: epididymitis $(\mathrm{n}=2)$, testicular trauma $(\mathrm{n}=4)$ testicular abscess $(\mathrm{n}=1)$, scrotal abscess $(\mathrm{n}=1)$.

More precisely, among 4 patients diagnosed with testicular torsion one (19 yo) was finally found with incomplete torsion, two with complete torsion (24 and 27 yo) and the last with abscess due to testicular necrosis (22 yo). In all cases, the involved testicle was edematous, enlarged and painful on palpation. The cremasteric reflex was absent and no relief of pain upon elevation of scrotum was observed. Both cases with complete torsion were characterized by scrotal erythema and unilateral position of the testicle, while abscess due to testicular necrosis was accompanied by edema involving the entire scrotum and fever.

One additional testicular abscess case, as a result of epididymitis no response to the medical treatment, was also diagnosed in a 65 year-old diabetic male. Edema involving the entire scrotum and fever were present.

Five out the six cases of blunt testicular trauma were unilateral and isolated while the remaining one was bilateral. All were associated with motor vehicle accident. Two (28 and 44 yo) out of the six patients exhibited symptoms of extreme scrotal pain associated with nausea and vomiting and were finally discovered with major testicular trauma upon surgical exploration. The remaining patients who experienced mild scrotal pain were diagnosed with minimal testicular trauma. Physical examination revealed a swollen, severely tender testicle with a visible hematoma in all cases. Scrotal ecchymosis was present in two cases.

In all testicular torsion cases, both the baseline US examination and CEUS equally diagnosed the underlying pathology. While Power-Doppler showed lack of vascularization of the affected testes, contrastenhanced US confirmed the absence of macro- and microvascularization (Figure-1), but failed to add any relevant information in the study of complete testicular torsion. In chronic (missed) torsion peritesticular tissues displayed increased vascularity on CEUS (Figure-2), while, in the study of incomplete torsion, CEUS showed a clear difference in the degree of enhancement between the normal and affected side (Figure-3).

CEUS was proved more accurate in the investigation of major testicular trauma, providing essential information on the viability of the affected organ. On conventional US, intra-parenchymal lacerations were visualized as linear or deliquescent non echogenic lesions. Only one scrotal sonogram showed a fractured testis with a disrupted tunica albuginea and testicular contents surrounded by tunica vaginalis, while in the remaining surgically treated testes $(n=2)$ the conventional US failed to clearly determine the presence and extend of tunica albuginea's eruption. When examined with contrast-enhanced technique surgically treated ruptured testes showed lack of enhancement 


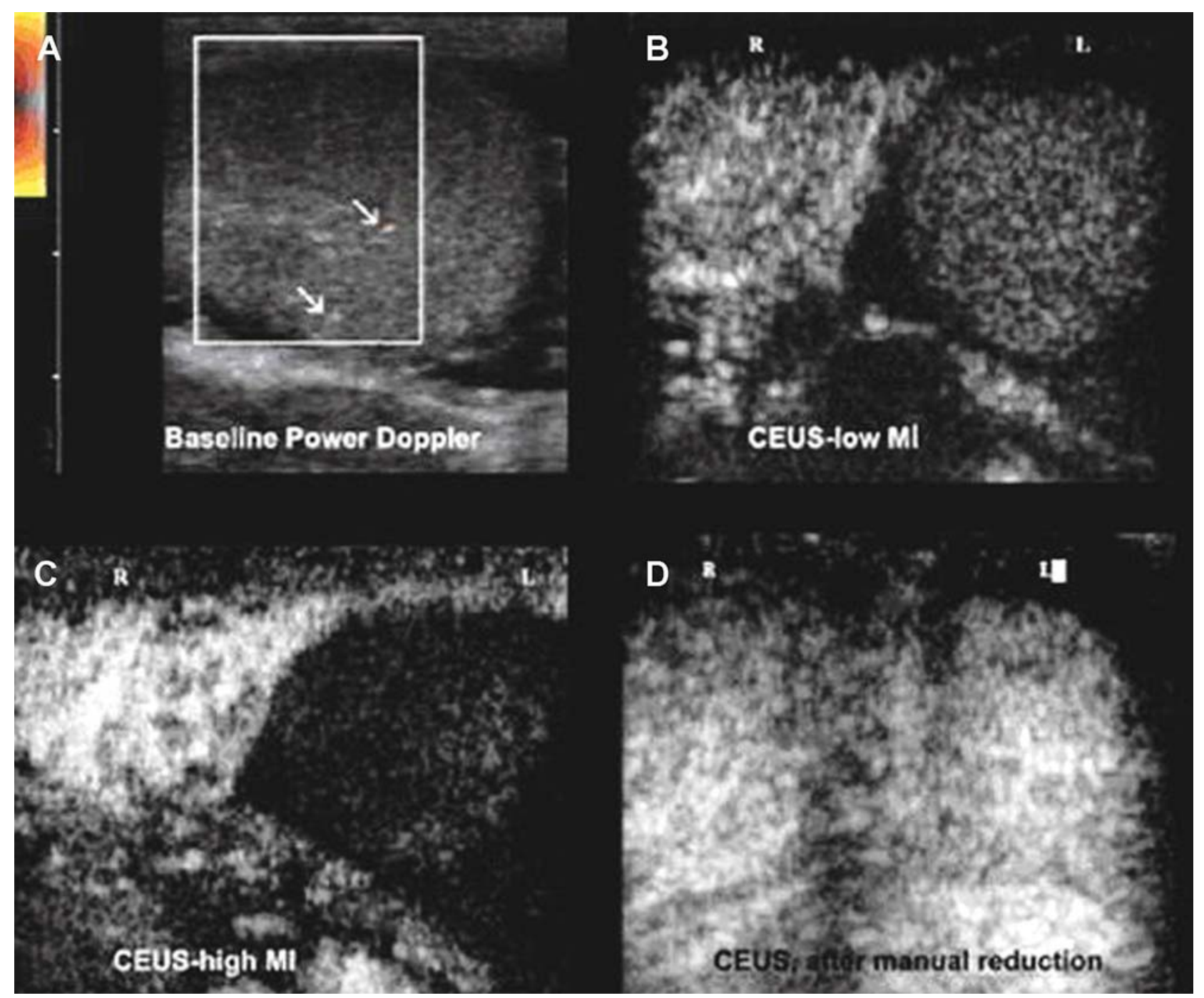

Figure l - Acute testicular torsion. A 27 year old male with acute left hemiscrotum. A) On initial power Doppler investigation of the left testis, only a few vascular signals (arrows) are detected (artifacts or incomplete torsion). B) On contrast-enhanced ultrasonography (CEUS) the left testis lacks enhancement and there is a clear difference when it is compared with the strong homogeneous enhancement of the normal side. C) The difference is more striking with the high MI (flash) technique. D) After reduction, CEUS demonstrates equal enhancement of the two testes.

in almost all their extent (Figure-4). On the contrary, conservatively treated testis showed a decreased, non-homogeneous, partial, contrast enhancement. Minimal testicular traumatic lesions were depicted as small hypoechoic vaguely dispersed areas, within a more echogenic normal testicular parenchyma which was not accompanied by a serious disorder of the testicular vascularity (Figure-5). Minimal traumatic injuries of the testis were almost uniformly presented as intratesticular hematoma in conventional US.
The conventional ultrasound diagnosis was in accordance with that of CEUS in the remaining positive cases; however, CEUS offered higher definition in the visualization of testis and scrotal abscess (Figures6 and 7). In all testicular abscess cases a peripheral, target like enhancement and evident central liquefaction was shown. In one out of the three cases with testicular abscess (a 69 yo diabetic patient), contrast enhanced US (52 sec from injection) also identified a multiple, echoic internal septae, with evident sep- 

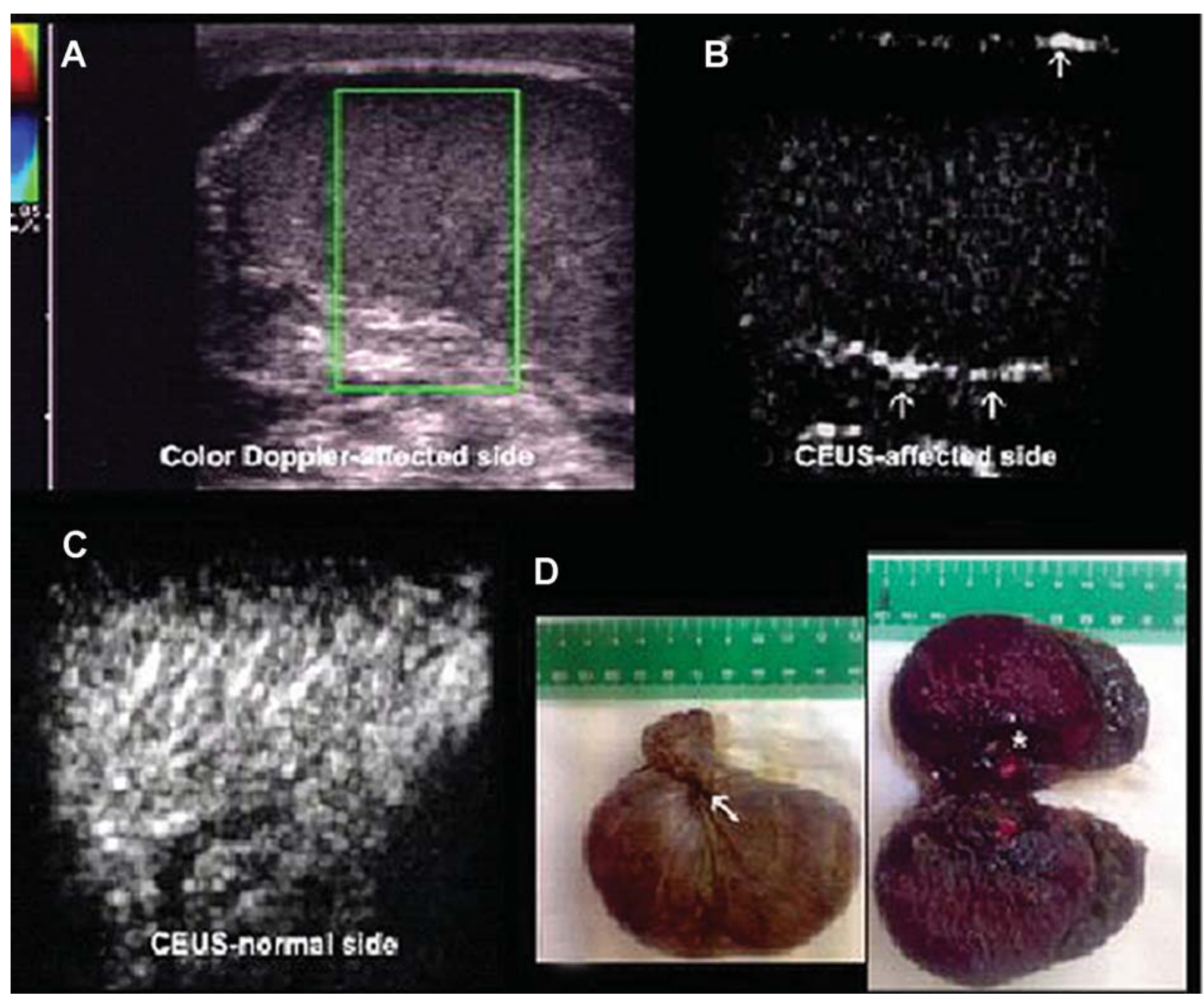

Figure 2 - Missed torsion. A 25-year-old patient presented with pain of the left hemiscrotum for 1 week. A) Baseline color Doppler shows an enlarged, slightly inhomogeneous left testis with no detectable flow. B) On contrast-enhanced ultrasonography (CEUS) the affected testis lacks enhancement and only enhancement of the peri-testicular tissues can be seen (arrows). C) The difference with the normal side is evident. D) The arrow indicates the site of torsion. There is also hemorrhagic necrosis $(*)$.

tal enhancement, which was missed in the baseline examination. Finally, CEUS ruled out the diagnosis of a tumor like small subcapsular hematoma, which mimicked a solid nodular lesion on baseline, where US demonstrated the absence of enhancement.

\section{COMMENTS}

In the late 90 's an experimental study by Coley et al., demonstrated contrast-enhanced US, even with the use of first-generation sonographic con- trast media, to be more accurate than color and power Doppler in the visualization of testis vascularity (8). Nevertheless, most researchers have mainly focused on the application of contrast-enhanced US to the study of focal renal or hepatic parenchymal lesions and no imaging studies of the scrotum using sonographic contrast media have been published over the last ten years. Recently, Catalano et al., using similar sonographic contrast media and technique with that of our study, evaluated the use of contrast-enhanced US in emergency radiology (7). Despite the large number of patients, only 8 out of the 126 cases investigated 


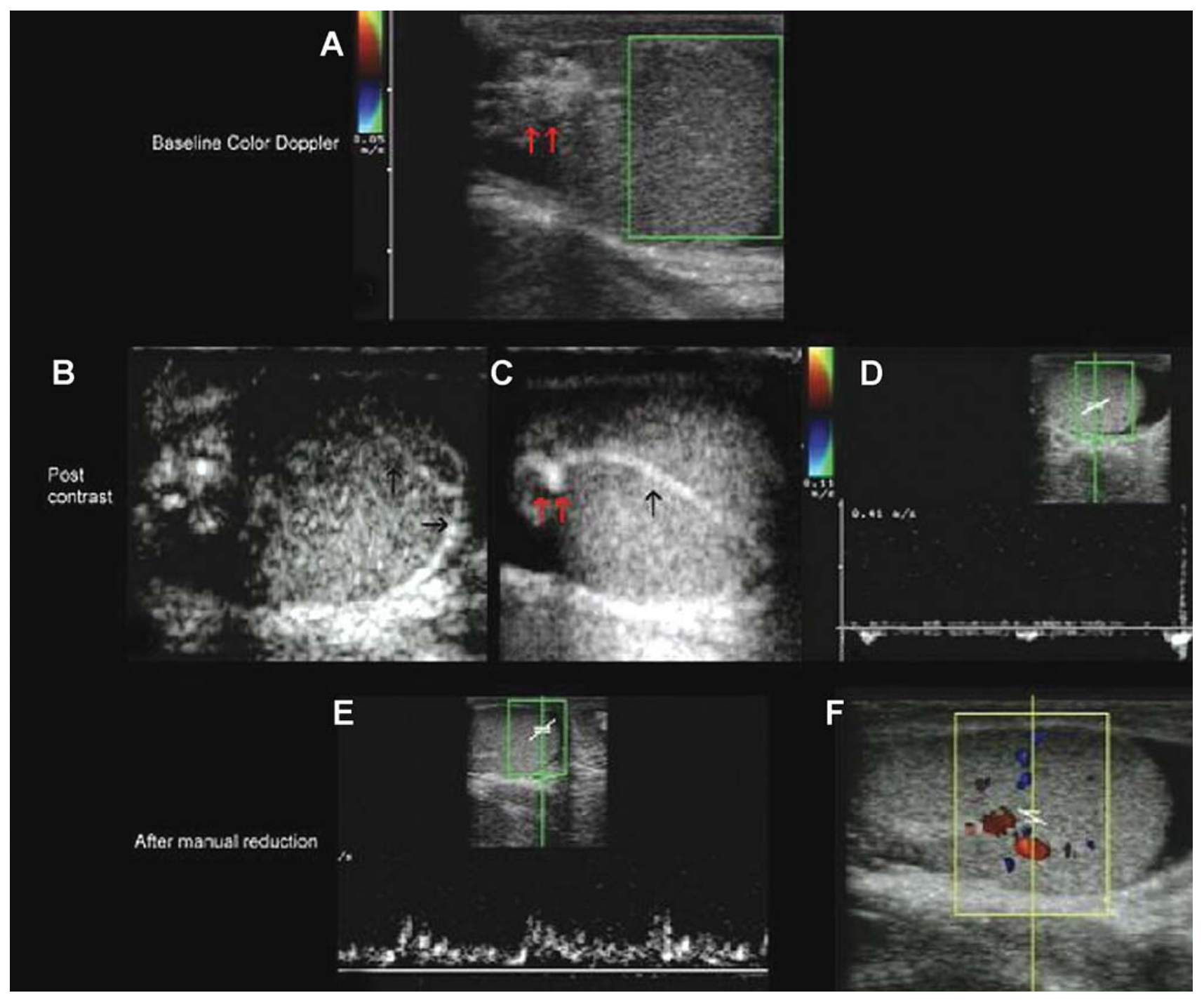

Figure 3 - Incomplete torsion. A 20-year-old man with sudden onset of left hemiscrotum pain 2 hours ago. A) Baseline color Doppler, shows no vascular signals within the testis. An ovoid inhomogeneous supratesticular mass that could represent a twisted spermatic cord (red arrows) is also evident. B) and C) contrast-enhanced ultrasonography (CEUS) reveals some patent intratesticular vessels (black arrows). One of them appears twisted above the testis (red arrows). D) Spectral analysis shortly after the administration of the echo-enhancer shows pathologic high resistance flow. E) After manual reduction intratesticular flow pattern has returned to normal. F) Multiple vascular signals are detected on color Doppler.

by the authors with the real-time contrast-specific US, concerned acute painful scrotum situations [testicular torsion $(n=6)$, epididymitis $(n=1)$, testicular trauma $(\mathrm{n}=4)]$. Although, no advantage over Doppler US has been shown it seems that real-time contrast-specific US is an effective technique in emergency imaging. Its role should not be considered as a replacement of Doppler US but as a useful integration of conventional US. In fact, compared to baseline sonography there is a loss of image quality with images appearing grainy, but this is widely compensated by the possibility of evaluating the area of interest in real time, which is impossible with intermittent-mode high mechanicalindex techniques $(10,11)$.

Our experience showed that CEUS fails to further increase the diagnostic accuracy of conventional grey scale and Doppler ultrasound in the investigation of testicular torsion; both Doppler US 


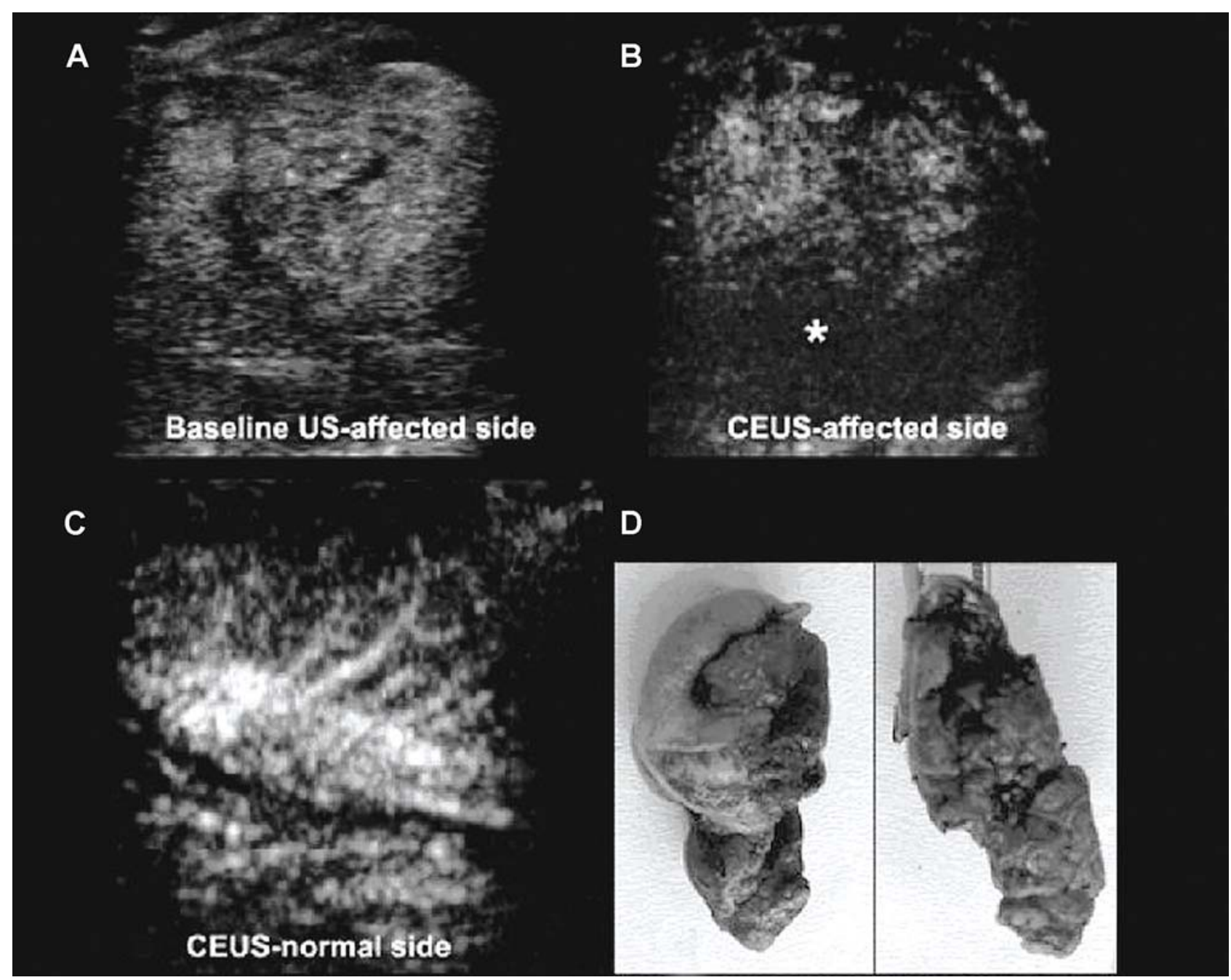

Figure 4 - Severe testicular trauma. A) On baseline ultrasound (US), the injured testis appears inhornogeneous, disorganized and with indistinct outline. B) On contrast-enhanced ultrasonography (CEUS), the same testis shows weak, inhornogeneous, patchy enhancement. The coexistent hematocele (*) lacks enhancement. C) Normal contralateral testis, for comparison. D) Parts of the shattered, resected testis.

and CEUS findings were equally in accordance with that of surgical exploration in patients with suspicious testicular torsion. It should be mentioned, however, that CEUS is not susceptible to artifacts that hinder a Color Doppler (and especially a Power Doppler) examination (11). For example, in incomplete, less than 360-degrees torsion cases the affected side often maintains some vascularity and comparison of color Doppler signals with the normal side can be difficult. In this case, CEUS can facilitate diagnosis by showing a clear difference in the degree of enhancement between the normal and affected side. Similarly, in chronic (missed) torsion, no testis enhancement on CEUS facilitates to interpret weak color Doppler signals when increased vascularity of the peri-testicular tissues occurs $(4,12,13)$. In addition, since CEUS requires no parameter optimization for the detection of slow flow, or flow in small vessels, it may prove easier and faster than color Doppler in the diagnosis of acute testicular torsion (14).

Detection of flow in testes of small children is another challenge for ultrasonography (13). Currently, the key clinical dilemma still remains to avoid surgery, especially in children, when clinical findings are 


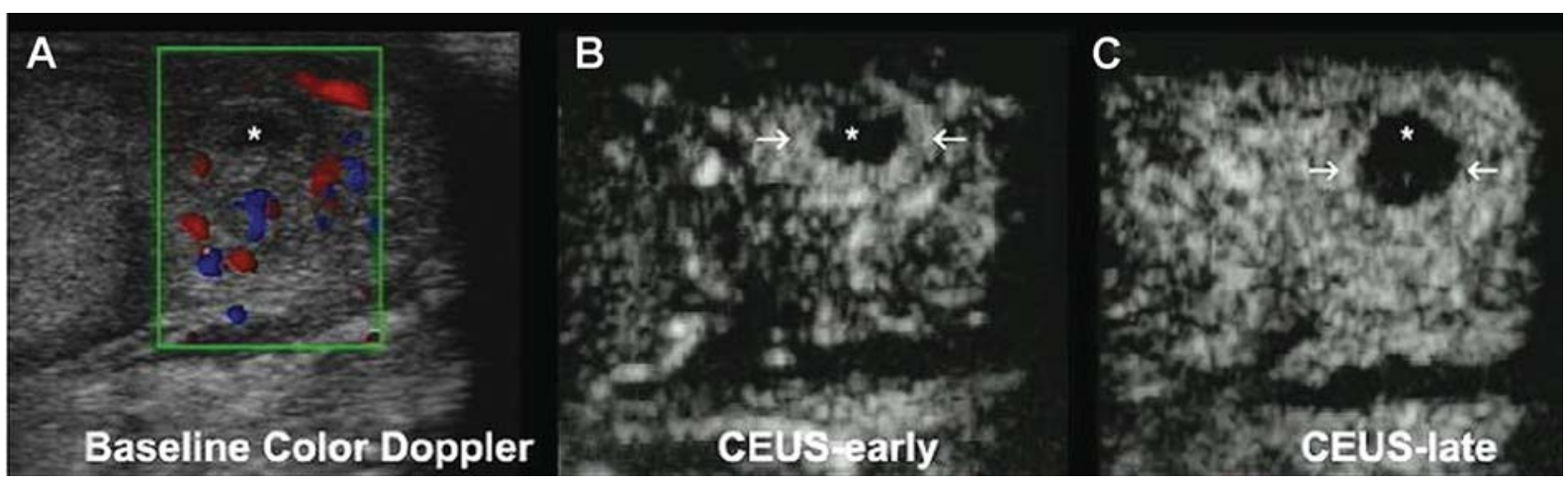

Figure 5-Epididymal abscess. A) Baseline color Doppler examination shows a round lesion with anechoic center (*), within an enlarged epididymal tail. Peripheral vascular signals are detected. B) Contrast-enhanced ultrasonography (CEUS), 15 seconds post injection shows early peripheral enhancement of the lesion (arrows). C) Thirty-eight seconds post injection, there is strong enhancement of the epididymal tail. The abscess shows typical rim enhancement (arrows) and non-enhancing center (*).

suspicious despite negative color Doppler ultrasound (CDU) results $(14,15)$. On the other hand, color Doppler may fail to demonstrate normal flow in testicles with a volume of less than $1 \mathrm{cc}$, leading thus to a false positive diagnosis of testicular torsion $(13,16)$. Therefore, CEUS as it is not susceptible to artifacts can, at least theoretically, facilitate the diagnosis of torsion in pediatric patients; however, the safety of SonoVue ${ }^{\circledR}$ in children has not been currently established.

Grayscale US findings of epididymo-orchits are enlarged hypoechoic epididymis and testis. These findings are non-specific and often are indistinguishable from testicular torsion (17). In contrast, combination of grey-scale US and color Doppler is helpful in evaluating patients with infection thus preventing unnecessary surgical exploration. Since US with color Doppler is effective in demonstrating the morphologic and hemodynamic changes in epididymitis and orchitis, CEUS does not seem to add to the diagnostic accuracy of the emergency ultrasound study. However, CEUS could play a role in the investigation of the complications of both epididymitis and orchitis. In
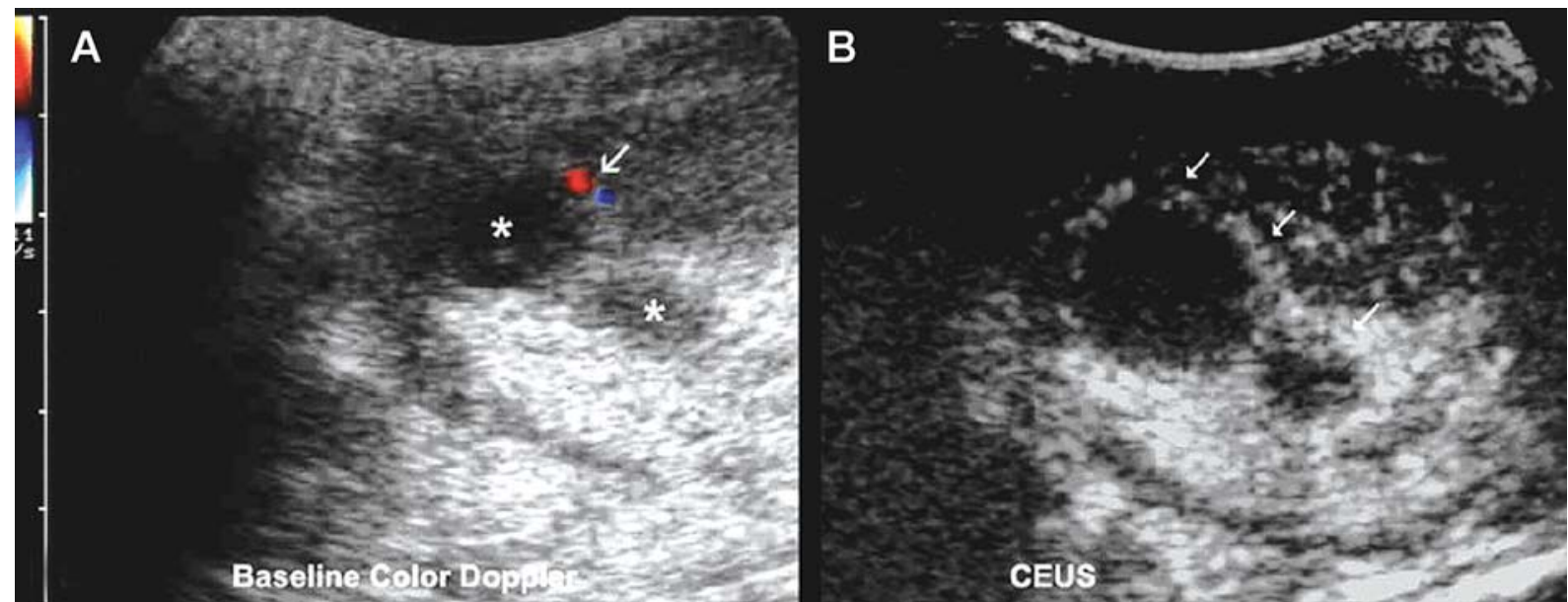

Figure 6 - Scrotal abscess. A) Baseline color Doppler of a sagittal section above the right testis. An inhomogeneous mass is demonstrated, that contains two ovoid, hypoechoic areas (*). A few peripheral vascular signals are detected (arrow). B) Contrast-enhanced ultrasonography (CEUS) of the same section. The hypoechoic areas exhibit peripheral rim enhancement and are more clearly delineated. The other parts of the mass show inhomogeneous enhancement. 


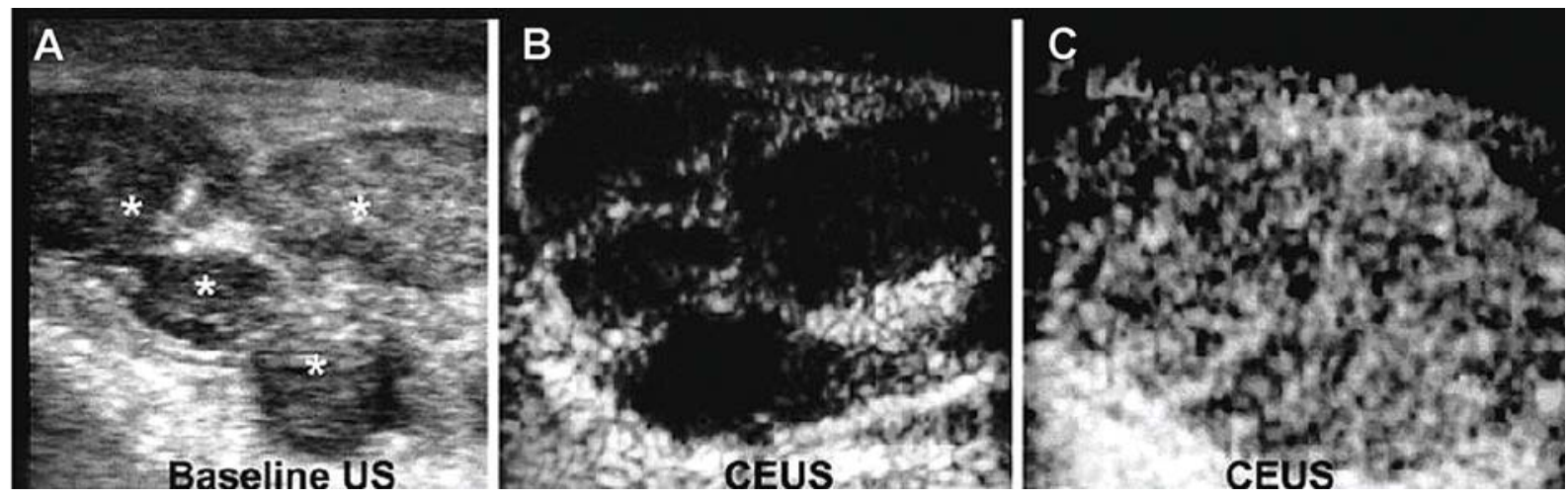

Figure 7 -Extratesticular hematoma. Patient with a painful lump at the upper part of the right hemiscrotum and history of closed scrotal injury 25 days ago. A) On baseline ultrasound US, a few ovoid hypoechoic structures (*) are demonstrated above the testis. B) On contrast-enhanced ultrasonography (CEUS) they lack enhancement. C) Normal enhancement of the ipsilateral testis for comparison.

fact, advanced epididymo-orchitis may cause testicular infarction as a result of extrinsic compression of testicular vascular supply by enlarged epididymis and spermatic cord and pyocele therefore; intratesticular vascularity is decreased (2). In addition, severe epididymo-orchitis may also cause testicular abscess and scrotal abscess, which is demonstrated as a complex echoic mass. These findings suggest the need for surgical exploration. In contrast, CEUS can confirm or exclude the presence of abscess in cases of serious, persistent inflammation (epididymitis or epididymoorchitis) $(12,17)$. In fact, CEUS findings are diagnostic: epididymal, testicular and scrotal abscesses show a typical strong rim enhancement and non-enhancing contents while enhancing septa may be demonstrated within the lesions (18). Additional information offered by CEUS may alter the management or outcome of patients with scrotal abscess permitting urologists to avoid surgery, especially in ageing patients with other significant life-limiting medical conditions. In our study, a left orchiectomy was performed in the patient with scrotal abscess and testicular torsion, while scrotal abscess in patient with epididymitis was managed by incision and drainage.

According to the perspective of the authors CEUS could be a useful complement to clinical and baseline sonographic examination, when it is not clear whether a traumatized scrotum requires surgical exploration (when, for example, a hematocele without an obvious testicular rupture is demonstrated). When compared with conventional ultrasound CEUS was proved more accurate in the investigation of major testicular trauma, providing essential information on the viability of the affected organ. In fact, the intensity and homogeneity of testicular perfusion on CEUS correlates with the severity of the trauma. Minor trauma (in the form of small contusions) usually causes no significant enhancement deficits. On the other hand, severely fractured testicles show weak, inhomogeneous, or patchy enhancement. Complete lack of enhancement may be seen in trauma-associated torsion or in cases of completely disorganized testicles. Similarly, hematomas (intra- or extra-testicular) and hematoceles show no enhancement. Therefore, CEUS improves their delineation and the differential diagnosis from other focal lesions. To our knowledge if rupture of the testis is detected sonographically, immediate surgical exploration is indicated. In contrast, sonographically detected hematoceles without visible signs of rupture should be treated conservatively. Since inappropriately protracted expectant management promotes testicular infection, atrophy, and necrosis, adequate preoperative diagnosis is essential for the viability of wounded testis (19). In our study, two out of 6 cases with testicular trauma were finally found with minimal lesion in CEUS and thus avoided unnecessary surgery.

Based on the above described findings, the possible indications for the CEUS investigation of acute scrotum can be summarized as follows: sus- 
pected testicular torsion, when color Doppler findings are equivocal (due to artifacts, or incomplete torsion), inflammation of the contents of the scrotum, when a complication is suspected, and scrotal trauma, in order to confirm a testicular damage that requires surgical intervention.

Two important limitations of CEUS should be underlined: as the low spatial resolution of the method, compared to baseline US and the additional cost of the contrast-agent (5). Nevertheless, it should be mentioned that CEUS is no more than an effective complement to grey-scale and color Doppler ultrasonography, therefore it is mandatory to perform a detailed initial examination of the scrotum with conventional US, in order not to miss subtle findings that may not be detected on the following CEUS examination. As regards the cost of the contrastagent it can be reduced by injecting a part of the standard dose. In our study, only $2.4 \mathrm{~mL}$ (i.e. half of the standard dose) of the echo-enhancer were used, and provided satisfactory tissue enhancement for at least 3 minutes. It is probable that even smaller doses can guarantee an accurate diagnostic examination. According to Catalano et al., an important concern regarding the use of CEUS is the additional time needed for the contrast-agent preparation, administration and performance of contrast-enhanced US scan (5). Our limited experience however has shown that CEUS of the scrotum can easily be accomplished in the emergency department, immediately after the baseline sonographic examination, and with a minimum delay (approx. $5 \mathrm{~min}$.) in the diagnostic work-up of the patient. Such a delay is insignificant in the investigation of acute scrotum, as it does not seem to alter the patient outcome.

\section{CONCLUSION}

Although current experience on the use of sonographic contrast media in the imaging study of acute painful scrotum is limited, it seems that CEUS can be used supplementary to traditional Doppler US in the investigation of blunt testicular trauma especially when uncertainty in diagnosis after appropriate clinical and radiographic evaluations occurs. In several selected acute scrotum cases, real-time contrast-specific ultrasound may also increase the efficacy of ultrasonographic diagnosis. The use of second-generation sonographic contrast media along with the newly-introduced low mechanical index software allows dynamic exploration of organ perfusion, with identification of both the macrocirculation and the lesion microcirculation. Therefore, CEUS can constitute a valuable integration of conventional grey-scale and color Doppler ultrasonography, particularly when these yield unclear, poorly visible findings. Further studies are required to clearly define the indications of this method.

\section{CONFLICT OF INTEREST}

None declared.

\section{REFERENCES}

1. Deurdulian C, Mittelstaedt CA, Chong WK, Fielding JR: US of acute scrotal trauma: optimal technique, imaging findings, and management. Radiographics. 2007; 27: 357-69.

2. Pavlica P, Barozzi L: Imaging of the acute scrotum. Eur Radiol. 2001; 11: 220-8.

3. Dogra VS, Gottlieb RH, Oka M, Rubens DJ: Sonography of the scrotum. Radiology. 2003; 227: 18-36.

4. Steinhardt GF, Boyarsky S, Mackey R: Testicular torsion: pitfalls of color Doppler sonography. J Urol. 1993; 150: 461-2.

5. Watanabe Y, Dohke M, Ohkubo K, Ishimori T, Amoh Y, Okumura A, et al.: Scrotal disorders: evaluation of testicular enhancement patterns at dynamic contrastenhanced subtraction MR imaging. Radiology. 2000; 217: 219-27.

6. Paltiel HJ, Connolly LP, Atala A, Paltiel AD, Zurakowski D, Treves ST: Acute scrotal symptoms in boys with an indeterminate clinical presentation: comparison of color Doppler sonography and scintigraphy. Radiology. 1998; 207: 223-31.

7. Catalano O, Lobianco R, Sandomenico F, Mattace Raso M, Siani A: Real-time, contrast-enhanced sonographic imaging in emergency radiology. Radiol Med. 2004; 108: 454-69.

8. Coley BD, Frush DP, Babcock DS, O'Hara SM, Lewis AG, Gelfand MJ, et al.: Acute testicular torsion: comparison of unenhanced and contrast-enhanced power 
Doppler US, color Doppler US, and radionuclide imaging. Radiology. 1996; 199: 441-6.

9. Liang RX, Xue ES, Lin LW, Yu L, Chen S, Yu LY, et al.: Correlation between sonographic appearance of experimental testicular ischemia and histological changes of the testis after reperfusion. Zhonghua Nan Ke Xue. 2009; 15: 115-21.

10. Lencioni R, Cioni D, Bartolozzi C: Tissue harmonic and contrast-specific imaging: back to gray scale in ultrasound. Eur Radiol. 2002; 12: 151-65.

11. Bauer A, Solbiati L, Weissman N: Ultrasound imaging with SonoVue: low mechanical index real-time imaging. Acad Radiol. 2002; 9 (Suppl 2): S282-4.

12. Berman JM, Beidle TR, Kunberger LE, Letourneau JG: Sonographic evaluation of acute intrascrotal pathology. AJR Am J Roentgenol. 1996; 166: 857-61.

13. Aso C, Enríquez G, Fité M, Torán N, Piró C, Piqueras J, et al.: Gray-scale and color Doppler sonography of scrotal disorders in children: an update. Radiographics. 2005; 25: 1197-214.

\author{
Correspondence address: \\ Dr. Stamatiou Konstantinos \\ Department of Urology \\ General Hospital of Pireas "Tzaneio" \\ 2 Salepoula str. \\ 18536 Piraeus, Greece \\ Fax: + 302 1042-96987 \\ E-mail: stamatiouk@gmail.com
}

\section{EDITORIAL COMMENT}

The paper by Moschouris et al. describes their experience using contrast-enhanced ultrasound (CEUS) to augment Doppler US (US) in the evaluation of 19 cases of acute scrotum. In cases of suspected torsion, CEUS provided no additional benefit beyond DUS. The authors state that CEUS provides improved images of vascular flow and may have a role in cases
14. Pepe P, Panella P, Pennisi M, Aragona F: Does color Doppler sonography improve the clinical assessment of patients with acute scrotum? Eur J Radiol. 2006; 60: $120-4$.

15. Luker GD, Siegel MJ: Color Doppler sonography of the scrotum in children. AJR Am J Roentgenol. 1994; 163: 649-55.

16. Bader TR, Kammerhuber F, Herneth AM: Testicular blood flow in boys as assessed at color Doppler and power Doppler sonography. Radiology. 1997; 202: 559-64. Erratum in: Radiology 1997; 203: 580.

17. Feld R, Middleton WD: Recent advances in sonography of the testis and scrotum. Radiol Clin North Am. 1992; 30: 1033-51.

18. Muttarak M, Lojanapiwat B: The painful scrotum: an ultrasonographical approach to diagnosis. Singapore Med J. 2005; 46: 352-7.

19. Buckley JC, McAninch JW: Use of ultrasonography for the diagnosis of testicular injuries in blunt scrotal trauma. J Urol. 2006; 175: 175-8.

Accepted after revision: July 27, 2009

of incomplete torsion or when the DUS is equivocal, but they do acknowledge that based on the current evidence, CEUS does not have a clinical role in the evaluation of suspected torsion. In cases of testicular or scrotal abscess, the authors again state that CEUS may provide better delineation of abscess from inflamed parenchyma, but with only 3 cases of abscess (none of 
which were diagnosed solely by CEUS) they do not have evidence to support that claim. Finally, in cases of testicular trauma that authors state that CEUS may be more specific in ruling out testicular rupture than conventional US, stating that in 2 of their cases unnecessary surgery was avoided by re-assuring CEUS findings when US was inconclusive. However, recent evidence suggests that magnetic resonance imaging (MRI) is very accurate in cases of testicular trauma when US is inconclusive (1), and is warranted when considering expectant management of a patient with possible testicular rupture. Furthermore, while avoiding surgical exploration of the scrotum is a laudable goal, the more critical issue in testicular trauma is identifying testicular rupture, and for this purpose conventional US is very sensitive (2). If future larger

\section{EDITORIAL COMMENT}

I would like congratulate Moschouris $\mathrm{H}$ et al. for this beautiful study. This study does have its limitations but also opens up new opportunities for research in scrotal pathology.

There is a need to improve the sensitivity and specificity of traditional ultrasound. These experimental imaging modalities include contrast-enhanced ultrasound, dynamic contrast magnetic resonance (MR), and near-infrared (NIR) imaging. Particular interest lies in the evaluation of pediatric testicular torsion.

The sensitivity and specificity of the current ultrasound machines is excellent in detecting absence of blood flow in testicular torsion patients and may not require ultrasound contrast enhancement in its evaluation. Color flow Doppler alone has a sensitivity of $86 \%$, specificity of $100 \%$, and accuracy of $97 \%$ in diagnosing testicular torsion (1). Blood flow within the testis can be quantified using spectral Doppler studies demonstrate that CEUS is as accurate as MRI in cases of testicular trauma, then it may have a role as a more cost-effective alternative, but at this time, CEUS does not have a clinical role in evaluation of the acute scrotum.

\section{REFERENCES}

1. Kim SH, Park S, Choi SH, Jeong WK, Choi JH: The efficacy of magnetic resonance imaging for the diagnosis of testicular rupture: a prospective preliminary study. J Trauma. 2009; 66: 239-42.

2. Guichard G, El Ammari J, Del Coro C, Cellarier D, Loock PY, Chabannes E, et al.: Accuracy of ultrasonography in diagnosis of testicular rupture after blunt scrotal trauma. Urology. 2008; 71: 52-6.

\section{Dr. Jeffrey Tiemstra Associate Professor Department of Family Medicine UIC College of Medicine Chicago, Illinois, USA E-mail:jtiemstr@uic.edu}

(2). Color Doppler sonography often has difficulty demonstrating perfusion within the normal pediatric testis. While still in its infancy, contrast-enhanced ultrasound has the potential to provide improved sensitivity in detecting flow in the pediatric testis (3). In an experimental study using 35 rabbits, Paltiel et al. (4) demonstrated that contrast-enhanced pulse-inversion sonography could reliably detect altered levels of testicular perfusion when compared to radiolabeled microsphere perfusion measurements.

Vasculitis involving the testes is uncommon, but may be seen in patients with polyarteritis nodosa $(3,4)$ and systemic lupus erythematosus $(5)$. Color Doppler evaluation may reveal the absence of testicular blood flow, mimicking torsion (5). It is in these patients with vasculitis where blood flow is so small that its beyond the resolution of the ultrasound machine to detect it that ultrasound contrast enhancement may play a significant role in their diagnosis $(6,7)$. 
Additionally, contrast enhanced sonography has a definite role in scrotal examinations, especially for characterizing small tumors less than two centimeters in size. Ultrasound contrast agents can play a significant role in testicular trauma to identify testicular contusions or hematomas and exclude testicular masses by demonstrating the presence of blood flow in them.

Near infrared fluorescence (NIRF) has also been performed with the intravenous administration of indocyanine green. In one preliminary study, NIRF was able to reliably detect vascular flow obstruction within the torsed testis of an adult male SpragueDawley rat model [Personal communication with VS Dogra et al. Abstract presented at World Congress of Endourology, Cleveland 2006]. This method can improve the sensitivity in detecting early testicular torsion and may increase testis salvage rate. As future investigations are completed, NIR spectroscopy and fluorescence will likely become a fast and cost-effective method in the initial evaluation of testicular torsion in the emergency setting.

\section{REFERENCES}

1. Burks DD, Markey BJ, Burkhard TK, Balsara ZN, Haluszka MM, Canning DA: Suspected testicular

\section{EDITORIAL COMMENT}

The aim of the study is very interesting, but several points must be implemented and improved.

The number of patients is too low; with the particular reference to the patients submitted to surgical exploration because of suspicious testicular torsion (four cases). The key clinical dilemma remains to avoid surgery, especially in children, when clinical findings are suspicious despite negative color Doppler ultrasound (CDU) results. Although CEUS evaluation after medium administration seems to be useful and accurate to detect intraparenchimal vascularization, a more consistent number of patients is needed. CEUS torsion and ischemia: evaluation with color Doppler sonography. Radiology. 1990; 175: 815-21.

2. Scoutt LM, Zawin ML, Taylor KJ: Doppler US. Part II. Clinical applications. Radiology. 1990; 174: 309-19.

3. Coley BD, Frush DP, Babcock DS, O'Hara SM, Lewis AG, Gelfand MJ, et al.: Acute testicular torsion: comparison of unenhanced and contrast-enhanced power Doppler US, color Doppler US, and radionuclide imaging. Radiology. 1996; 199: 441-6.

4. Paltiel HJ, Kalish LA, Susaeta RA, Frauscher F, O'Kane PL, Freitas-Filho LG: Pulse-inversion US imaging of testicular ischemia: quantitative and qualitative analyses in a rabbit model. Radiology. 2006; 239 : 718-29.

5. Suty JM, Hubert J, Duquenne M, Weryha G, Mangin P: Bilateral testicular ischemia in vasculitis. Differential diagnosis with torsion and the value of color Doppler ultrasonography. Prog Urol. 1995; 5: 586-9.

6. Leibovici D, Strauss S, Sharon A: Acute, painful, swollen testis in polyarteritis nodosa: a diagnostic problem. Harefuah. 1999; 136: 938-9.

7. Dogra VS, Bhatt S, Rubens DJ: Sonographic evaluation of testicular torsion. Ultrasound Clin. 2008: 1; 55-66.

Dr. Vikram S. Dogra

Department of Imaging Sciences

University of Rochester School of Medicine

Rochester, New York, USA

E-mail:vikram_dogra@urmc.rochester.edu

evaluation, as testicular scintigraphy, introduces a quantitative parameter and could improve CDU that in most cases is an operator depending procedure. In conclusion, an excellent idea supported (until now) by an anecdotal series that needs further studies.

Dr. Pietro Pepe

Department of Urology Cannizzaro Hospital

Catania, Italy

E-mail:piepepe@hotmail.com 


\section{EDITORIAL COMMENT}

This paper demonstrates the usefulness of contrast-enhanced ultrasonography in the diagnosis of acute scrotum by determining testicular perfusion based on contrast enhancement of testicles through the administration of microbubble contrast media.

Today, color and power Doppler ultrasonography (US) is the initial imaging modality used in the evaluation of symptomatic scrotum. It is readily available and noninvasive, and the testis itself is highly amenable to sonographic examination. However, scrotal US has some flaws and problems. Doppler US results are highly dependent on operators' skill, and detectability of blood flow depends on patient's age, testicular volume, and sensitivity of the US equipment used. Therefore, in a variety of clinical settings, inconclusive results of color and power Doppler US need another imaging method or modality for accurate and confirmatory assessment of scrotal disorders.

Use of contrast media in US seems to be a great idea because testicular perfusion can be much more conspicuous with contrast-enhanced US than only with Doppler US, as reported in this article. In magnetic resonance imaging (MRI), Watanabe et al. (reference \#5) first showed the usefulness of contrastenhanced imaging in the evaluation of acute scrotum.
With use of contrast agents, US is expected to provide as accurate qualitative information about testicular blood flow as MRI.

The difference between this new contrast-enhanced US and MRI could be semi-quantitative analysis of testicular perfusion. MRI imaging performs dynamic contrast-enhanced examination, which allows for semi-quantitative assessment of blood flow. Though US could not provide semi-quantitative information, US can be more convenient and easier to be performed in the emergency clinical settings than MRI.

As the authors described, this new method can be robust and useful to differentiate testicular torsion from other pathologies such as acute epididymitis which can be treated with antibiotics conservatively. In blunt trauma, this method could provide accurate information about the severity of testicular damage, rupture of tunica albuginea, and the presence or absence of hematoma. This method would also have a potential to characterize testicular neoplasm.

In the near future, contrast-enhanced US could hopefully be performed as the initial and robust imaging modality by the urologists and radiologists on the front line of medical care.

Dr. Yuji Watanabe

Radiologist-in-Chief Department of Radiology Kurashiki Central Hospital Miwa, Kurashiki, Japan E-mail:yw5904@kchnet.or.jp 